11,12

\title{
Термодинамические и магнитные свойства трехвершинной модели Поттса на треугольной решетке с учетом взаимодействия вторых ближайших соседей
}

\author{
(C) А.Б. Бабаев ${ }^{1,2}$, Т.Р. Ризванова ${ }^{1}$, А.К. Муртазаев ${ }^{1,3}$ \\ ${ }^{1}$ Институт фризики им. Х.И. Амирханова ДагНЦ РАН, \\ Махачкала, Россия \\ ${ }^{2}$ Дагестанский государственный педагогический университет, \\ Махачкала, Россия \\ ${ }^{3}$ Дагестанский государственный университет, \\ Махачкала, Россия \\ E-mail: b_albert78@mail.ru
}

(Поступила в Редакцию 21 марта 2017 г.)

На основе метода Монте-Карло изучены термодинамические и магнитные свойства в двумерных структурах описываемых трехвершинной моделью Поттса на треугольной решетке с учетом первых и вторых ближайших соседей с величинами взаимодействия $J_{1}$ и $J_{2}$ соответственно. На основе анализа термодинамических параметров теплоемкости, параметра порядка, восприимчивости и кумулянтов Биндера четвертого порядка, показано, что в трехвершинной модели Поттса с величинами взаимодействия $J_{1}>0$ и $J_{2}<0$ в диапазоне значений $0 \leq|r| \leq 1 / 3, r=J_{2} / J_{1}$ наблюдается фазовый переход второго рода.

Работа поддержана грантом РФФИ № 16-02-00214.

DOI: 10.21883/FTT.2017.12.45242.084

В последнее двадцатилетие интенсивно обсуждаются фазовые переходы (ФП) и критические явления (КЯ) в магнетиках, описываемых двумерными (2D) решеточными моделями Изинга и Поттса [1-4]. Это связано с тем, что низко-размерные решеточные модели описывают большой класс реальных физических систем: слоистые магнетики, пленки жидкого гелия, сверхпроводящие пленки, адсорбированные пленки и др. [5,6]. Антиферромагнетик на треугольной решетке является примером фрустрированной спиновой системы. В качестве примера геометрической фрустрации можно привести антиферромагнитную модель Изинга на треугольной решетке с учетом взаимодействия только ближайших соседей. В ней невозможно, расположить все спины так, чтобы каждая пара взаимодействующих соседей была антипараллельна (рис. $1, a)$. Из рис. $1, a$ может показаться, что фрустрация возможна только на треугольных решетках, однако это не так. На рис. $1, b$ приведена трехвершинная антиферромагнитная (АФ) модель Поттса с учетом взаимодействия только первых ближайших соседей на той же треугольной решетке. Как видно из рисунка, при учете только ближайших соседей магнитная система будет упорядоченной при конечной температуре и фрустрации отсутствуют. При учете вторых ближайших соседей конкуренция обменных взаимодействий может привести к фрустрациям, т. е. такому пространственному расположению магнитных моментов атомов в кристалле, при котором невозможно одновременное антиферромагнитное упорядочение всех взаимодействующих спинов (см. рис. $1, b)$. Эффекты фрустраций играют важную роль в различных магнитных системах. Экспериментальные [7] и теоретические исследования [8] позволили установить, что фрустрированные системы проявляют свойства, отличные от соответствующих нефрустрированных систем.

Двумерная трехвершинная модель Поттса на треугольной решетке для случая $J_{1}<0$ и $J_{2}<0$ нами исследовалась в работе $[9,10]$. В этих работах на основе анализа энтропии, теплоемкости, и кумулянтов Биндера четвертого порядка было показано, что при $0<r<0.2$ и $1.0<r<2.0$ наблюдаются фазовые переходы первого рода. В то время как в интервале $0.2 \leq r \leq 1.0$ фазовый переход отсутствует, и наблюдаются фрустрации. Случай $J_{1}>0$ и $J_{2}<0$ был рассмотрен в работах $[10,11]$ и было показано, что фрустрации в этом случае возникают в интервале изменений величины $0.5<|r|<1.0$. Поведение термодинамических и магнитных параметров при ФП в
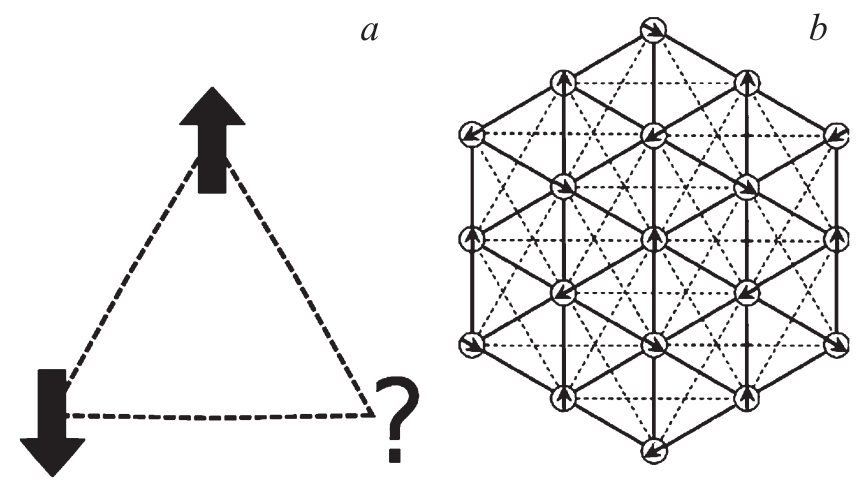

Рис. 1. Фрустрации в модели Изинга (a), трехвершинная модель Поттса на треугольной решетке $(b)$. 
этих работах не были изучены. Исследование особенностей термодинамических и магнитных параметров при ФП второго рода в 2D трехвершинной модели Поттса на треугольной решетке является главной задачей этой работы.

Трехвершинная $(q=3)$ модель Поттса на треугольной решетке с учетом взаимодействия вторых ближайших соседей (см. рис. $1, b)$ описывается следующим микроскопическим гамильтонианом [5]:

$$
H=-J_{1} \sum_{i, j} \cos \theta_{i, j}-J_{2} \sum_{i, k} \cos \theta_{i, k},
$$

где $J_{1}$ и $J_{2}-$ параметры обменных ферро- $\left(J_{1}>0\right)$ и антиферромагнитного $\left(J_{2}<0\right)$ взаимодействия для ближайших и вторых ближайших соседей соответственно. $\theta_{i, j}, \theta_{i, k}-$ углы между взаимодействующими спинами $S_{i}-S_{j}$ и $S_{i}-S_{k}$ соответственно. Модель Поттса с гамильтонианом вида (1) также хорошо описывает физические свойства многих неупорядоченных сруктур (см. $[12,13])$.

Расчеты проведены для систем с периодическими граничными условиями и с линейными размерами $L \times L=N, L=18 \div 54$. При этом отношение обменного взаимодействия вторых и ближайших соседей менялось в интервале $0 \leq|r| \leq 1.0$, где $|r| \leq J_{2} / J_{1}$. Кроме того, нами проведен анализ поведения термодинамических и магнитных параметров, определены температуры ФП и вычислены критические температуры. Начальные конфигурации задавались таким образом, чтобы все спины находились в разных состояниях. Для вывода системы в равновесное состояние вычислялось время релаксации $\tau_{0}$ для всех систем с линейными размерами $L$. Затем усреднение проводилось по участку марковской цепи длиной $\tau=200 \tau_{0}$. Кроме того, для повышения точности расчетов проводилось усреднение по 10 различным начальным конфигурациям. Затем эти данные использовались для расчета средних значений термодинамических параметров.

Для наблюдения за температурным ходом поведения теплоемкости и восприимчивости нами использовались флуктуационные соотношения [14]

$$
\begin{gathered}
C=\left(N K^{2}\right)\left(\left\langle U^{2}\right\rangle-\langle U\rangle^{2}\right), \\
\chi=(N K)\left(\left\langle m^{2}\right\rangle-\langle m\rangle^{2}\right),
\end{gathered}
$$

где $K=|J| / k_{\mathrm{B}} T, N=L^{2}-$ число узлов, $U-$ внутренняя энергия, $m-$ параметр порядка. В качестве параметра порядка $m$ для ферро- $\left(m_{\mathrm{F}}\right)$ и антиферромагнитной $\left(m_{\mathrm{AF}}\right)$ модели Поттса использовались следующие выражения [15]:

$$
\begin{gathered}
m_{\mathrm{F}}=\left\langle\frac{3}{2} \sum_{\alpha=1}^{3}\left(\frac{N_{\alpha}}{N}-\frac{1}{3}\right)^{2}\right\rangle^{1 / 2}, \\
m_{\mathrm{AF}}=\frac{3}{2}\left\langle\sum_{\alpha \neq \beta \neq \gamma}\left(\frac{N_{\alpha}+N_{\beta}+N_{\gamma}}{N}-\frac{1}{3}\right)^{2}\right\rangle^{1 / 2},
\end{gathered}
$$

где $N_{\alpha}=\left\{N_{1}, N_{2}, N_{3}\right\}, N_{1}$ - число спинов в состоянии с $q=1, N_{2}$ - число спинов в состоянии с $q=2$,

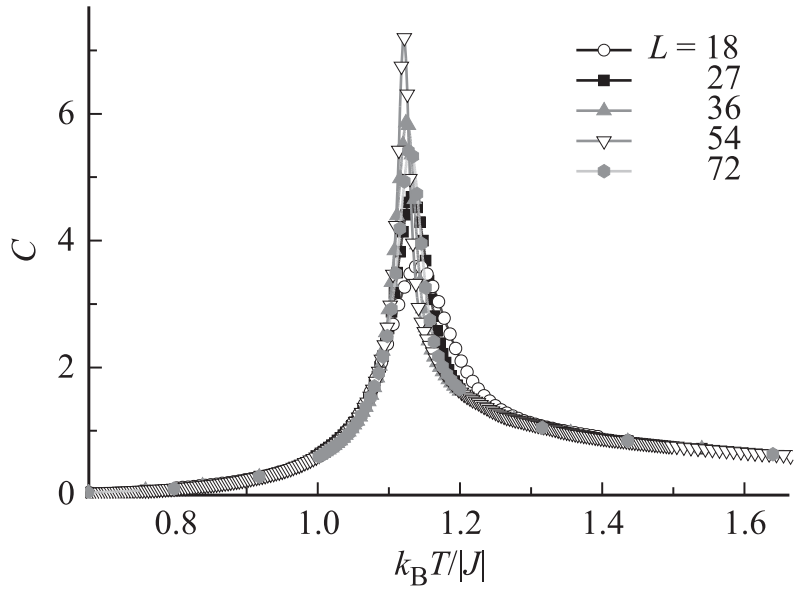

Рис. 2. Температурная зависимость теплоемкости $C$ трехвершинной модели Поттса с величинами взаимодействий $J_{1}>0$ и $J_{2}<0$ при $|r|=0.333$.

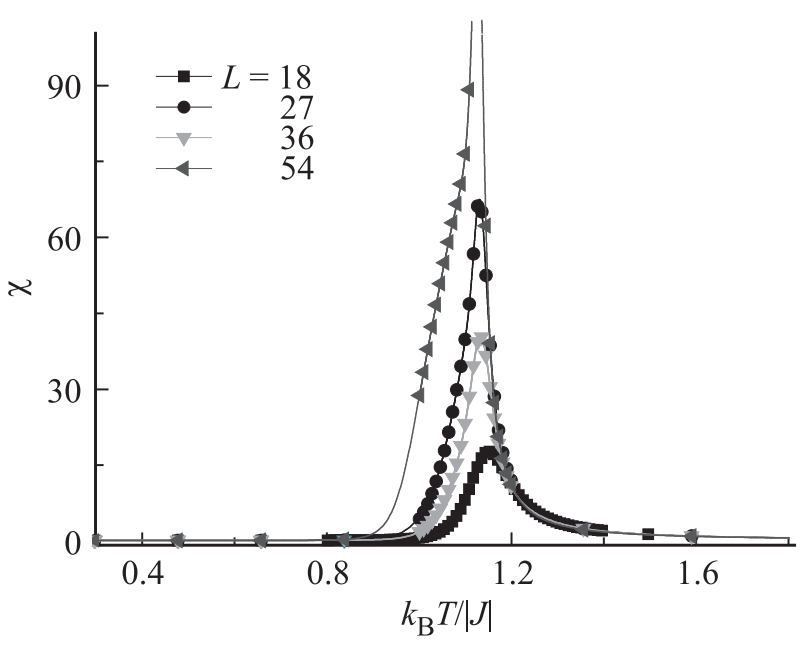

Рис. 3. Температурная зависимость восприимчивости $\chi$ трехвершинной модели Поттса с величинами взаимодействий $J_{1}>0$ и $J_{2}<0$ при $|r|=0.333$.

$N_{3}$ - число спинов в состоянии с $q=3, N_{\alpha}, N_{\beta}, N_{\gamma}$ число спинов в подрешетке $A, B$ и $C$ соответственно, $N=L^{2}$. Угловые скобки означают термодинамическое усреднение.

На рис. 2 и 3 представлены характерные зависимости теплоемкости и восприимчивости для двумерной трехвершинной модели Поттса на треугольной решетке с величинами взаимодействия $J_{1}>0$ и $J_{2}<0$ при $|r|=1 / 3$. Здесь и далее на всех рисунках погрешность данных не превосходит размеры символов, используемых для построения графиков. Отметим, что в зависимостях теплоемкости $C$ и восприимчивости $\chi$ от температуры для всех исследуемых нами систем проявляются четко выраженные максимумы, и эти максимумы в пределах погрешности приходятся на одну и ту же температуру. Такое же поведение для теплоемкости и восприимчивости наблюдалось для систем в интервале 


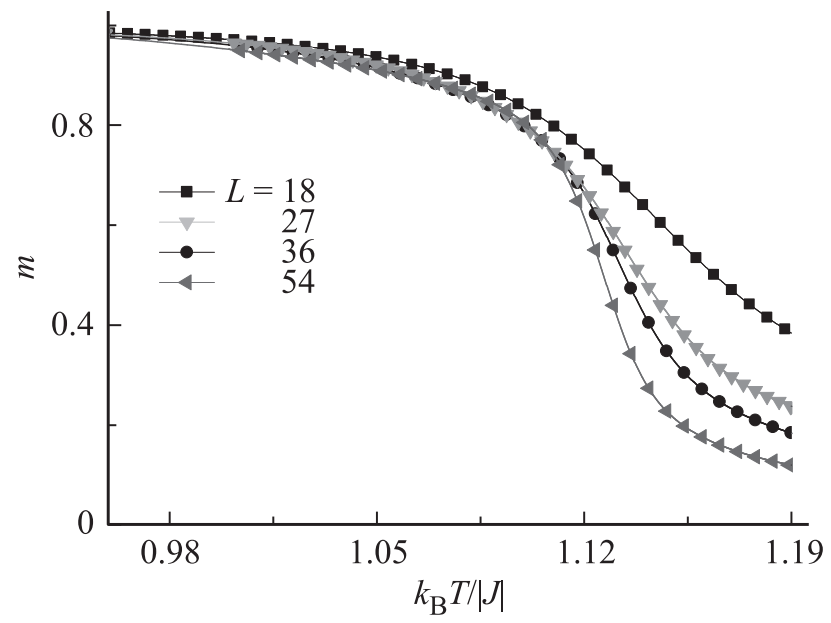

Рис. 4. Температурная зависимость параметра порядка $m$ трехвершинной модели Поттса с величинами взаимодействий $J_{1}>0$ и $J_{2}<0$, при $|r|=1 / 3$.

изменения $0 \leq|r| \leq 1 / 3$. Вне этого диапазона изменений $r$, как нами было отмечено в работах $[10,11]$ проявляются эффекты, связанные с фрустрациями и ФП отсутствует. Аналогичное поведение в трехвершинной модели Поттса с величинами взаимодействий $J_{1}<0$ и $J_{2}<0$ наблюдалось в интервале изменений величины $0.2 \leq|r| \leq 1.0[9]$.

На рис. 4 представлены температурные зависимости параметра порядка $m$ для трехвершинной модели Поттса при $J_{1}>0, J_{2}<0$ и $|r|=1 / 3$ для систем с линейными размерами $L=18,27,36,54$. Как видно из рисунка, для всех рассмотренных систем с линейными размерами $L$ наблюдается монотонное уменьшение величины $m$ с увеличением температуры, что является характерным признаком ФП второго рода.

Для определения критических температур и анализа характера фазового перехода использовался метод кумулянтов Биндера четвертого порядка [16]

$$
\begin{gathered}
V_{L}(T)=1-\frac{\left\langle E^{4}\right\rangle_{L}}{3\left\langle E^{2}\right\rangle_{L}^{2}}, \\
U_{L}(T)=1-\frac{\left\langle m^{4}(T, L)\right\rangle_{L}}{3\left\langle m^{2}(T, L)\right\rangle_{L}^{2}},
\end{gathered}
$$

где $E-$ энергия и $m-$ параметр порядка системы с линейными размерами $L$. Выражения (6) и (7) позволяют с хорошей точностью определить $T_{c}$ при фазовых переходах первого и второго рода соответственно. Следует отметить, что применение кумулянтов Биндера позволяет также хорошо тестировать род фазового перехода в системе. Методика определения критической температуры нами подробно описана в работах $[17,18]$. Известно, что фазовые переходы второго рода характеризуются, в частности, следующими отличительными особенностями [7]: усредненная величина $V_{L}(T)$ стремится к тривиальному значению $V^{*}$ согласно выражению

$$
V(T)=V^{*}+b L^{-d}
$$

при $L \rightarrow \infty$ и $T=T_{c}(L)$, где $V^{*}=2 / 3$, что и продемонстрировано на рис. 5. Кроме того, в случае ФП второго рода кривые температурной зависимости кумулянтов Биндера $U_{L}(T)$ по параметру порядка $\mathrm{m}$ имеют четко выраженную точку пересечения. Характерные зависимости кумулянтов Биндера $U_{L}(T)$ для 2D модели Поттса от температуры для систем с разными линейными размерами $L$ приведены на рис. 6. Как видно из рис. 6 в критической области наблюдается четко выраженная точка пересечения, что и свидетельствует о ФП второго рода. Кроме того, этот рисунок демонстрирует, насколько точно можно определить критическую температуру $T_{c}$.

Таким образом, в трехвершинной модели Поттса с величинами взаимодействий $J_{1}>0$ и $J_{2}<0$ в интервале

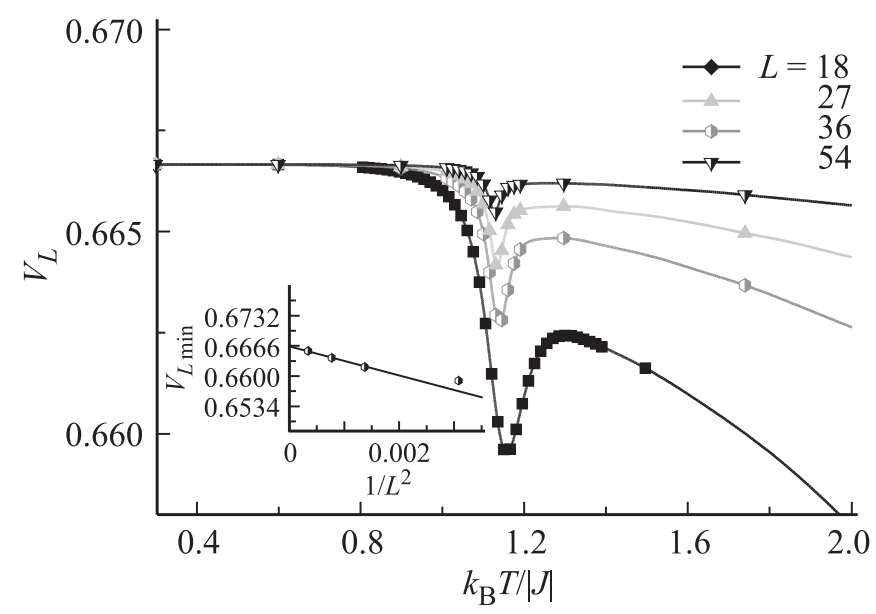

Рис. 5. Температурная зависимость кумулянтов Биндера $V_{L}(T)$ для двумерной модели Поттса с величинами взаимодействий $J_{1}>0$ и $J_{2}<0$, при $|r|=1 / 3$. На вставке приведена аппроксимация зависимостей $V_{L, \min }$ от $L$.

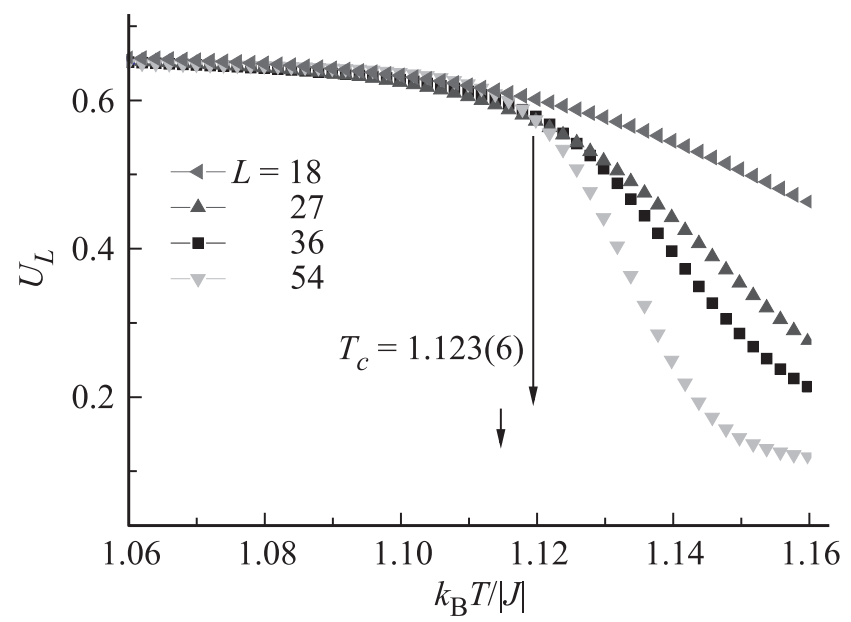

Рис. 6. Температурная зависимость кумулянтов Биндера $U_{L}(T)$ для трехвершинной модели Поттса с величинами взаимодействий $J_{1}>0$ и $J_{2}<0$, при $|r|=1 / 3$. 
изменений величины $0 \leq|r| \leq 1 / 3$ наблюдаются ФП второго рода. Вне этого интервала ФП отсутствует, что связано с вырождением основного состояния системы. Изучение значений $r$ из указанного диапазона имеет большое значение при создании различных синтетических метамагнетиков и выяснения процесса упорядочения для антиферромагнитных материалов с треугольной структурой $[2,8]$.

\section{Список литературы}

[1] H.T. Diep. Frustrated spin systems. World Scientific Publishing Co. Pte. Ltd., Singapore (2004).

[2] С.В. Малеев. УФН 172, 6, 617 (2002).

[3] А.К. Муртазаев, А.Б. Бабаев. Изв. РАН. Сер. физ. 77, 10, 1476 (2013).

[4] Л.Н. Щур. УФН 182, 7, 787 (2012).

[5] F.Y. Wu. Rev. Mod. Phys. 54, 235 (1982).

[6] W. Zhang, Y. Deng. Phys. Rev. E 78, 031103 (2008).

[7] D. Loison, K.D. Schotte. Eur. Phys. J. B 5, 735 (1998).

[8] Р.С. Гехт. УФН 159, 261 (1989).

[9] А.К. Муртазаев, А.Б. Бабаев, М.А. Магомедов, Ф.А. Кассан-Оглы, А.И. Прошкин. Письма в ЖЭТФ 100, 267 (2014). [JETP Lett. 100, 242 (2014)].

[10] А.Б. Бабаев, М.А. Магомедов, А.К. Муртазаев, Ф.А. Кассан-Оглы, А.И. Прошкин. ЖЭТФ 149, 357 (2016). [JЕТР 122, 310 (2016)].

[11] А.Б. Бабаев, А.К. Муртазаев, Э.М. Сулейманов, Т.Р. Ризванова. ФТТ 58, 10, 2001 (2016).

[12] A.K. Murtazaev, A.B. Babaev. JETP 115, 6, 1042 (2012).

[13] А.К. Муртазаев, А.Б. Бабаев, Г.Я. Атаева. Физика низких температур. 39, 2, 194 (2013). [Low Temperature Physics 39, 2, 147 (2013)].

[14] P. Peczac, A.M. Ferrenberg, D.P. Landau. Phys. Rev. B 43, 6087 (1991).

[15] Y. Saito. J. Phys. A 15, 1885 (1982).

[16] K. Eichhorn, K. Binder. J. Phys.: Condens. Matter 8, 5209 (1996).

[17] А.К. Муртазаев, А.Б. Бабаев. ЖЭТФ 143, 1, 116 (2013). [JETP 116, 1, 101 (2013)].

[18] А.К. Муртазаев, А.Б. Бабаев. Письма в ЖЭТФ 99, 9, 618 (2014). [JETP Lett. 99, 9, 535 (2014)]. 